\title{
Local Stress Engineering for the Optimization of p-GaN Gate HEMTs Power Devices
}

\author{
T. Cosnier, L. Lucci and A. Torrès \\ CEA LETI, Minatec Campus \\ Grenoble, France \\ Email: thibault.cosnier@cea.fr
}

\author{
M. Pala \\ C2N, CNRS, Université Paris-Saclay \\ Orsay, France \\ Email: marco.pala@c2n.upsaclay.fr
}

\begin{abstract}
The impact of the built-in stress of the SiN passivation layer on $\mathbf{p}-\mathrm{GaN}$ gate High Electron Mobility transistors (HEMTs) is investigated through TCAD simulations. Local modifications of electron confinement in the channel area due to stressor deposition can be exploited to increase the threshold voltage independently of the $\mathrm{ON}$-state resistance (up to $+1.5 \mathrm{~V}$ for $\mathrm{t}_{\mathrm{SiN}}=200 \mathrm{~nm}, \sigma_{\mathrm{SiN}}=-2 \mathrm{GPa}$ and $\left.\mathrm{L}_{\mathrm{G}}=0.2 \mu \mathrm{m}\right)$. This technique can also be easily combined with an AIGaN backbarrier structure to increase the design margin of $\mathrm{p}-\mathrm{GaN}$ gate normally-OFF HEMTs.
\end{abstract}

Keywords-p-GaN gate HEMT, stress engineering, passivation layer, normally-off, threshold voltage, TCAD simulation

\section{INTRODUCTION}

Owing to their superior material properties, GaN-based high electron mobility transistors (HEMTs) are seen as promising candidates for next generation power switching applications. High-density and high-mobility 2-D electron gas (2DEG) induced at the $\mathrm{AlGaN} / \mathrm{GaN}$ heterointerface by spontaneous and piezoelectric polarization fields leads to normally-ON type HEMTs [1]. Today, most efforts are oriented toward the development of normally-OFF type devices, required for failsafe operation and circuit complexity reduction.

Silicon nitride ( $\mathrm{SiN}$ ) passivation is largely employed in the fabrication process of typical AlGaN/GaN HEMTs to resolve surface state issues, suppress current collapse and improve overall device performance [2]. It has been recently proposed that local relaxation of unintentionally stressed $\mathrm{SiN}$ passivation can directly alter the threshold voltage $\left(\mathrm{V}_{\mathrm{TH}}\right)$ of HEMTs through piezoelectric polarization-induced charge density generation in the gate area [3], [4]. By adjusting the plasmaenhanced chemical vapor deposition (PECVD) conditions of $\mathrm{SiN}$, the amplitude and the sign of the intrinsic stress of the film can be controlled [5]. Although normally-OFF devices based solely on SiN stress effect may be designed, they would require agressive scaling of the parameters (stress, thickness) of the film as well as using ultra-short gate lengths, such that this may not be experimentally realistic [6].

On the other hand, several approaches have been introduced to suppress the $2 \mathrm{DEG}$ underneath the gate region to achieve a positive threshold voltage $\left(\mathrm{V}_{\mathrm{TH}}\right)$, such as recessing the $\mathrm{AlGaN}$ barrier [7] or using fluorine-based plasma treatments [8]. A different technique involves growing a $\mathrm{p}-\mathrm{GaN}$ or $\mathrm{p}$ AlGaN layer on top of the barrier to deplete the 2DEG [9].
While p-type GaN gate technology provides secure normallyoff operation, it lacks of design margin to improve the tradeoff faced between $\mathrm{V}_{\mathrm{TH}}$ and the ON-state resistance $\left(\mathrm{R}_{\mathrm{ON}}\right)$. Employing an AlGaN back-barrier can help to increase channel confinement and $\mathrm{V}_{\mathrm{TH}}$ [10] but it also partly depletes the 2DEG in the access regions, thus increasing $R_{O N}$. Hence, finding new techniques to increase $V_{T H}$ independently of $R_{\mathrm{ON}}$ is an essential challenge in the development of $\mathrm{p}-\mathrm{GaN}$ gate normally-OFF HEMTs.

The purpose of this work is to provide trends for the optimization of $\mathrm{p}-\mathrm{GaN}$ gate HEMTs using stressed films. We demonstrate that the mechanical interaction of compressive films can be used to generate a negative polarization-induced charge density in the gate area, locally increasing the electron confinement. This leads to the achievement of a better tradeoff between $V_{\mathrm{TH}}$ and $\mathrm{R}_{\mathrm{ON}}$, where $\mathrm{V}_{\mathrm{TH}}$ can be increased independently of $R_{\mathrm{ON}}$. Furthermore, we also show that this technique can be easily combined with an $\mathrm{AlGaN}$ back-barrier for larger design margin.

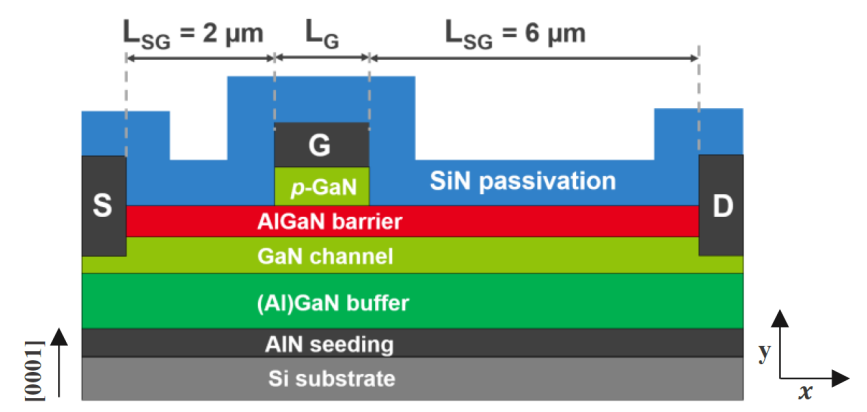

Fig. 1. Illustrative cross section plot (out of $x-y$ scale) of the p-GaN gate HEMT considered in this study. Gate patterning and ohmic contact formation precede the $\mathrm{SiN}$ deposition.

\section{Structure and Simulation Methodology}

\section{A. Device Structure}

We consider the p-GaN gate HEMT structure shown in Figure 1 comprising a $2 \mu \mathrm{m}(\mathrm{Al}) \mathrm{GaN}$ buffer, $30 \mathrm{~nm} \mathrm{GaN}$ channel, $15 \mathrm{~nm} \mathrm{Al}_{0.20} \mathrm{Ga}_{0.80} \mathrm{~N}$ barrier and $100 \mathrm{~nm}$-type $\mathrm{GaN}$ gate with $3 \times 10^{17} \mathrm{~cm}^{-3} \mathrm{Mg}$ acceptors density. TiN gate metal thickness is $30 \mathrm{~nm}$ and $\mathrm{SiN}$ film thickness $\left(\mathrm{t}_{\mathrm{SiN}}\right)$ is set to $200 \mathrm{~nm}$. Gate length $\left(\mathrm{L}_{\mathrm{G}}\right)$ is varied between 0.2 and $0.5 \mu \mathrm{m}$. 


\section{B. Simulation Methodology}

A three-step simulation methodology shown in Figure 2 is employed to assess the impact of the stressed layer on the electrical characteristics of the $\mathrm{p}-\mathrm{GaN}$ gate HEMT.

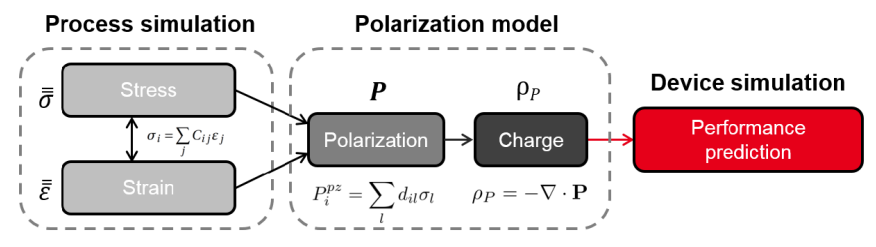

Fig. 2. Three-step simulation framework methodology used to predict the impact of the mechanical relaxation of the stressed passivation film on device characteristics.

1) Process Simulation: First, the mechanical state of the structure is determined by modeling device fabrication using Sentaurus Process 2D simulations [11]. The intrinsic stress of the passivation layer $\left(\sigma_{\mathrm{SiN}}\right)$ is varied from $-2 \mathrm{GPa}$ compressive to $+2 \mathrm{GPa}$ tensile to reflect achievable experimental range [5]. A multi-layer deposition scheme consisting in 20 deposition steps is employed for accurate modeling of the mechanical impact of SiN film in the gate region [12].

2) Polarization Model: Then, the total polarization $P$ in the III-N layers is calculated by summing the contributions of spontaneous and piezoelectric polarizations as $P=P_{s p}+P_{p z}$.

Piezoelectric polarization is obtained from stress tensor $\sigma$ and piezoelectric coefficients $d$ as $P_{p z}=d \cdot \sigma$ in the matrixbased formulation. Polarization-induced fixed charge density $\rho_{\mathrm{P}}$ arising from the spatial divergence of the polarization in the III-N layers is calculated as $\rho_{P}=-\nabla \cdot P$

3) Device Simulation: Device simulations are performed using Sentaurus Device [13]. Fixed polarization-induced charge density is included in anisotropic Poisson equation and typical models used in the simulation of GaN-based HEMTs [14] as well as standard parameters for III-N materials [15] are employed.

\section{RESUlTS AND DISCUSSION}

\section{A. Lateral Stress}

The impact of the elastic stress relaxation of $\mathrm{SiN}$ on lateral stress $\left(\sigma_{x x}\right)$ is depicted in Figure 3 for $\mathrm{L}_{\mathrm{G}}=0.20 \mu \mathrm{m}$. For unstrained $\mathrm{SiN}$, the $\mathrm{AlGaN}$ layer is under constant tensile epitaxial stress and $\mathrm{GaN}$ layers are relaxed. However, nonuniform additional compressive (tensile) lateral stress is induced in the gate area in III-N layers due to compressive (tensile) built-in film stress and adds up to the initial stress.

\section{B. Polarization-Induced Charge Density}

The distribution of polarization-induced charge density $\left(\rho_{\mathrm{P}}\right)$ created in the gate region for $\mathrm{L}_{\mathrm{G}}=0.2 \mu \mathrm{m}$ is illustrated in Figure 4. Fixed negative charges in the range $10^{16}-10^{18} \mathrm{~cm}^{-3}$ are generated in the channel region and extend deeply in the $\mathrm{GaN}$ buffer. Moreover, SiN relaxation induces fixed positives charges to be created just outside the gate area.

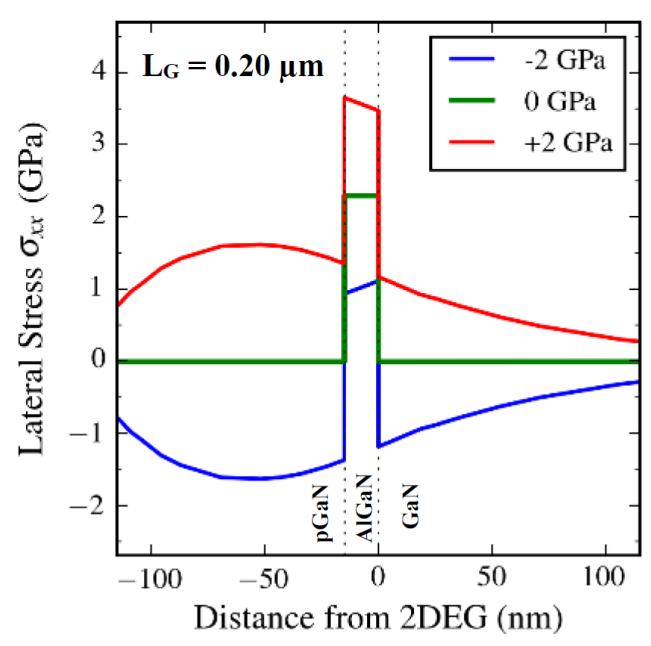

Fig. 3. Vertical profile of lateral stress $\left(\sigma_{x x}\right)$ along a cut performed at the center of the gate. The mechanical interaction of the stressed layer in the gate vicinity leads to non-uniform $\sigma_{x x}$ variation along the $c$-axis direction.

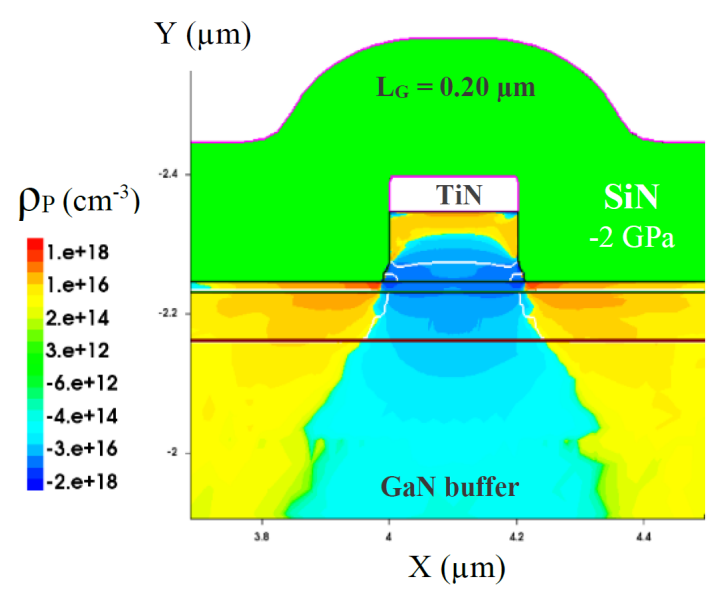

Fig. 4. Two-dimensional distribution of the polarization-induced charge density $\left(\rho_{\mathrm{P}}\right)$ generated in the III-N heterostructure consequently of stressed nitride layer deposition. $\sigma_{\mathrm{SiN}}=-2 \mathrm{GPa}, \mathrm{L}_{\mathrm{G}}=0.2 \mu \mathrm{m}$.

\section{Equilibrium Conduction Band Energy}

The electrostatic charges generated in the III-N layers affect the conduction band energy (CBE) level in the channel region as shown in Figures 5 and 6. Compared to the reference structure $\left(\sigma_{\mathrm{SiN}}=0 \mathrm{GPa}\right)$, the $\mathrm{CBE}$ is significantly lifted (lowered) by the effect of compressive (tensile) SiN stress. Results indicate that this shift of CBE is not limited to the $\mathrm{p}-\mathrm{GaN}$ gate but extends deeper in the GaN buffer.

The horizontal profile of the CBE along the 2DEG region displayed in Figure 6 clearly shows tremendous modification of the gate depletion region due to SiN stress. Compressive (tensile) SiN leads to a higher (lower) and wider (narrower) gate depletion region. Furthermore, for a same stress magnitude, $\sigma_{\mathrm{SiN}}>0$ leads to more significant changes of the CBE profile and almost cancels the $\mathrm{p}-\mathrm{GaN}$ gate induced potential barrier. 


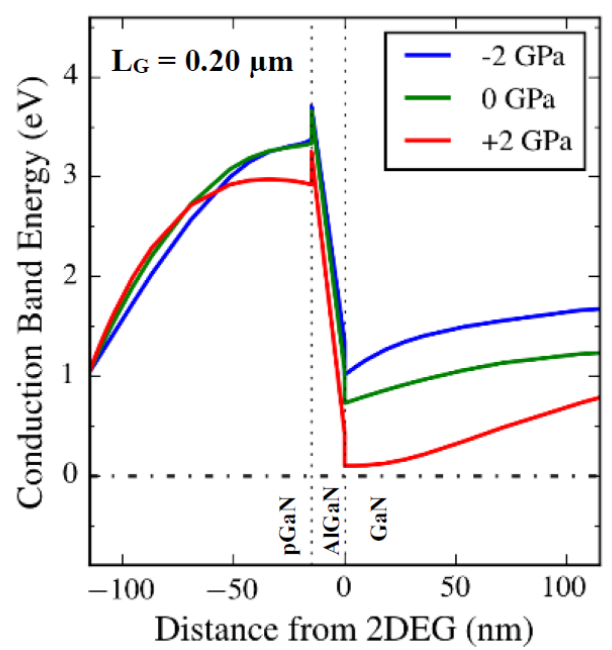

Fig. 5. Vertical profile of the equilibrium conduction band energy level $\left(\mathrm{V}_{\mathrm{GS}}=0 \mathrm{~V}, \mathrm{~V}_{\mathrm{DS}}=0 \mathrm{~V}\right)$, at the center of the gate, for varying $\sigma_{\mathrm{SiN}}$.

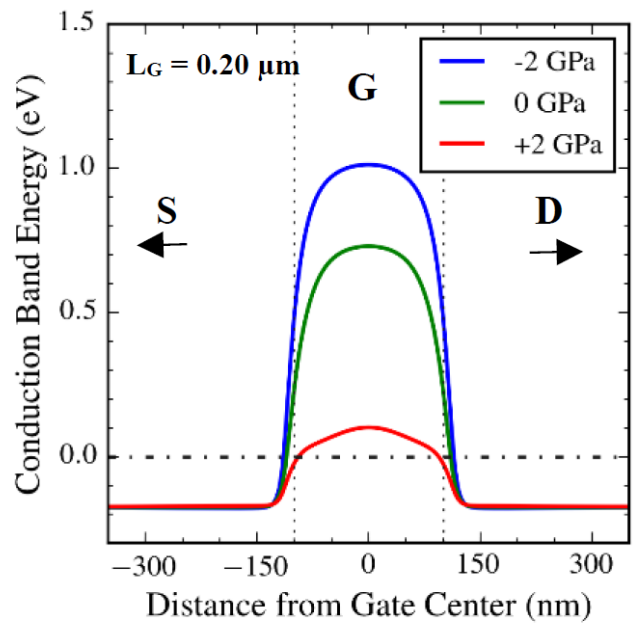

Fig. 6. Horizontal profile of the equilibrium conduction band energy level $\left(\mathrm{V}_{\mathrm{GS}}=0 \mathrm{~V}, \mathrm{~V}_{\mathrm{DS}}=0 \mathrm{~V}\right)$ along the AlGaN/GaN interface for varying $\sigma_{\mathrm{SiN}}$.

\section{Device Transfer Characteristics}

The simulated transfer characteristics of $\mathrm{p}-\mathrm{GaN}$ gate HEMTs with $\mathrm{L}_{\mathrm{G}}=0.2 \mu \mathrm{m}$ for different values of $\sigma_{\mathrm{SiN}}$ are shown in Figure 7. Consistently with the increased channel confinement discussed in Section III-C, the transfer characteristics exhibits a positive $\mathrm{V}_{\mathrm{TH}}$ shift when $-2 \mathrm{GPa}$ compressively strained $\mathrm{SiN}$ is deposited on top of the $\mathrm{p}-\mathrm{GaN}$ gate structure. Moreover, a slight increase in saturation current is observed. This is due to the positive polarization-induced charge density created outside the gate area.

Conversely, it is shown that the introduction of tensile $\mathrm{SiN}$ leads to a large negative threshold voltage shift such that the device is no longer normally-OFF. This is well explained by the critical degradation of the electron confinement observed in Figures 5 and 6. A slight decrease in saturation current

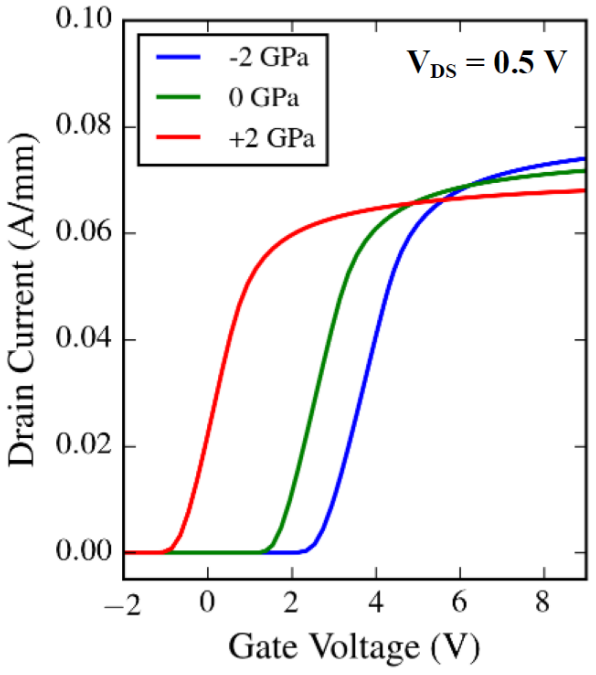

Fig. 7. Transfer characteristics $\mathrm{I}_{\mathrm{DS}}\left(\mathrm{V}_{\mathrm{GS}}\right)$ of the simulated $\mathrm{pGaN}$ gate HEMTs showing the impact of $\sigma_{\mathrm{SiN}}$. The device embedding highly tensile $\mathrm{SiN}$ film is no longer normally-OFF due to the large negative $\mathrm{V}_{\mathrm{TH}}$ shift.

is also observed due to negative polarization charge density created outsite the gate area.

\section{E. Impact of Gate Length on $\mathrm{V}_{\mathrm{TH}}$ Shift}

The gate length parameter plays a key role in quantifying the impact of $\mathrm{SiN}$ on device performances. Indeed, the interactions between the stress fields created in the III-N layers at both edges of the gate are magnified when the gate length is decreased. This is verified when plotting the evolution of the threshold voltage shift against intrinsic film stress in Figure 8. When $\mathrm{L}_{\mathrm{G}}$ decreases, the magnitude of $\Delta \mathrm{V}_{\mathrm{TH}}$ increases significantly. It is interesting to note that the trends are not symmetrical with respect to the sign of $\sigma_{\mathrm{SiN}}$. Indeed, for short gate lengths $\left(\mathrm{L}_{\mathrm{G}}<0.5 \mu \mathrm{m}\right)$, the magnitude of $\Delta \mathrm{V}_{\mathrm{TH}}$ is greater for tensile films than for compressive films. This is consistent with the evolution of the potential barrier observed in Section III-C. However this trend is reversed for longer gate lengths $\left(\mathrm{L}_{\mathrm{G}} \geq 0.5 \mu \mathrm{m}\right)$, where the magnitude of $\Delta \mathrm{V}_{\mathrm{TH}}$ is greater for $\sigma_{\mathrm{SiN}}<0$. This is due to the $\mathrm{CBE}$ increase at both gate edges whereas for $\sigma_{\mathrm{SiN}}>0$, the $\mathrm{CBE}$ is lower at gate edges but remains weakly impacted at gate center.

\section{F. Trade-off between $\mathrm{V}_{\mathrm{TH}}$ and $\mathrm{R}_{\mathrm{ON}}$}

In order to evaluate more extensively the benefits of local stress engineering, simulations combining an $\mathrm{AlGaN}$ buffer (or back-barrier) and intrinsically stressed SiN were carried out. The Al content of the buffer $\left(x_{\text {buffer }}\right)$ was changed from 0.00 to 0.07 and $\sigma_{\mathrm{SiN}}$ was ranged from $-2 \mathrm{GPa}$ to $+1 \mathrm{GPa}$. Obtained results are expressed in terms of the trade-off between the threshold voltage $\mathrm{V}_{\mathrm{TH}}$ and the specific on-state resistance $\mathrm{R}_{\mathrm{ON}}$ and displayed in Figure 9.

As compared to a reference $\mathrm{GaN}$ buffer device $\mathrm{R}$ with unstrained $\operatorname{SiN}\left(x_{\text {buffer }}=0.00, \sigma_{\mathrm{SiN}}=0 \mathrm{GPa}\right.$ ), devices with increasing $x_{\text {buffer }}$ show positive $\mathrm{V}_{\mathrm{TH}}$ shift and substantial 


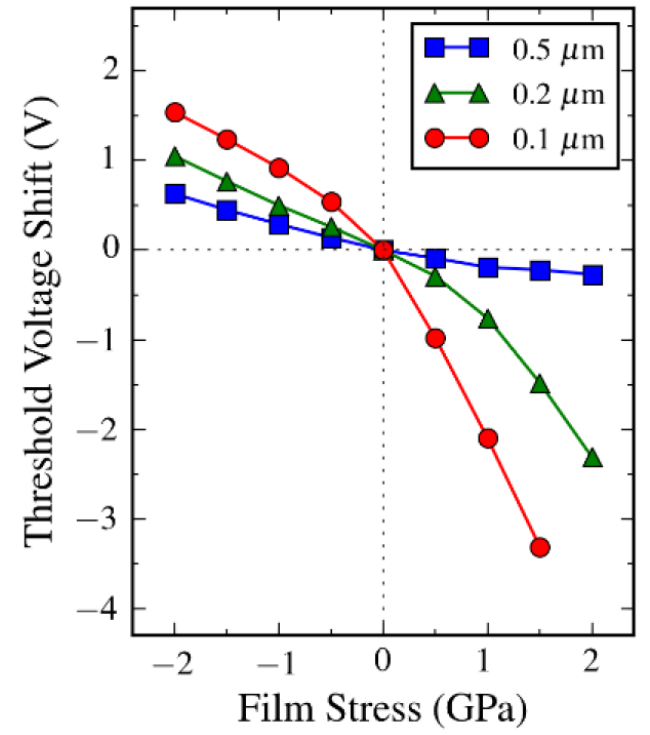

Fig. 8. Threshold voltage shift versus $\sigma_{\mathrm{SiN}}$ for varying $\mathrm{L}_{\mathrm{G}}$ values.

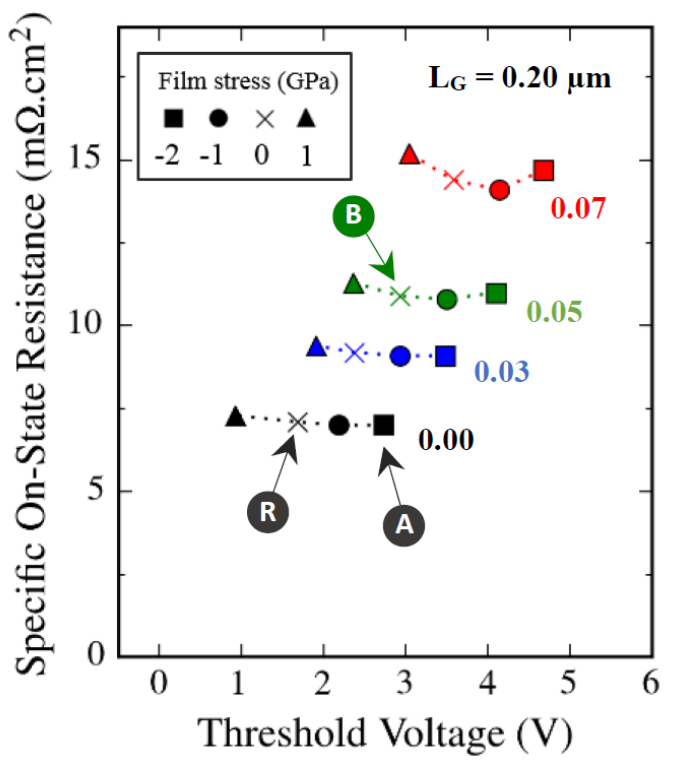

Fig. 9. Comparison of the trade-off between $\mathrm{V}_{\mathrm{TH}}$ and $\mathrm{R}_{\mathrm{ON}}$ for $\sigma_{\mathrm{SiN}}$ ranging from $-2 \mathrm{GPa}$ to $1 \mathrm{GPa}$ and $x_{\text {buffer }}$ ranging from 0.00 to 0.07 .

$\mathrm{R}_{\mathrm{ON}}$ increase, due to stronger confinement and partial depletion of the $2 \mathrm{DEG}$ in the access region. Thus, while device $\mathrm{B}$ $\left(x_{\text {buffer }}=0.05, \sigma_{\mathrm{SiN}}=0 \mathrm{GPa}\right)$ can increase $\mathrm{V}_{\mathrm{TH}}$ from $1.7 \mathrm{~V}$ to $3.0 \mathrm{~V}$, it also increases $\mathrm{R}_{\mathrm{ON}}$ by $\sim 70 \%$. On the other hand, device $\mathrm{A}\left(x_{\text {buffer }}=0.00, \sigma_{\mathrm{SiN}}=-2 \mathrm{GPa}\right)$ also increases $\mathrm{V}_{\mathrm{TH}}$ of the reference device by nearly the same amount but without deteriorating $R_{\mathrm{ON}}$. Therefore, local stress engineering of the p-GaN gate HEMT can increase $\mathrm{V}_{\mathrm{TH}}$ almost independently of $R_{\mathrm{ON}}$, which leads to a great improvement of the $V_{\mathrm{TH}}-\mathrm{R}_{\mathrm{ON}}$ trade-off. Moreover, this results clearly indicate that SiN stress effect can be incorporated in $\mathrm{p}-\mathrm{GaN}$ gate HEMTs employing AlGaN back-barrier structures to achieve better performances.
In this way, the combination of both techniques could e.g. be used to increase the $\mathrm{Al}$ content of the $\mathrm{AlGaN}$ barrier to reduce $R_{\mathrm{ON}}$ while maintaining $\mathrm{V}_{\mathrm{TH}}$ around a value of $2 \mathrm{~V}$.

\section{CONCLUSION}

This paper reveals the crucial role of stress engineering in obtaining high-performance $\mathrm{p}-\mathrm{GaN}$ gate normally-OFF HEMTs. While it is shown that tensile films deposited on top of the gate may lead to critical degradation of the safe operation, strategies employing compressively deposited thick films and relatively short gate lengths can be implemented to greatly improve the trade-off between the threshold voltage and the on-state resistance of the devices. Moreover, this technique can be used in conjunction with AlGaN backbarriers to furthermore increase the design margin.

\section{REFERENCES}

[1] O. Ambacher, B. Foutz, J. Smart, J. R. Shealy, N. G. Weimann, K. Chu, M. Murphy, A. J. Sierakowski, W. J. Schaff, L. F. Eastman, R. Dimitrov, A. Mitchell, and M. Stutzmann, "Two dimensional electron gases induced by spontaneous and piezoelectric polarization in undoped and doped $\mathrm{AlGaN} / \mathrm{GaN}$ heterostructures," Journal of Applied Physics, vol. 87, no. 1, pp. 334-344, Jan. 2000.

[2] W. Lu, V. Kumar, R. Schwindt, E. Piner, and I. Adesida, "A comparative study of surface passivation on AlGaN/GaN HEMTs," Solid-State Electronics, vol. 46, no. 9, pp. 1441-1444, Sep. 2002.

[3] E. T. Yu and P. M. Asbeck, "Local Polarization Effects in Nitride Heterostructures and Devices," in Polarization Effects in Semiconductors, D. C. Wood and D. Jena, Eds. Springer US, 2008, pp. 217-264.

[4] N. Shigekawa and S. Sugitani, "Numerical analysis of impact of stress in passivation films on electrical properties in $\mathrm{AlGaN} / \mathrm{GaN}$ heterostructures," IEICE Electronics Express, vol. 6, no. 14, pp. 1045-1050, 2009.

[5] P. Morin, G. Raymond, D. Benoit, P. Maury, and R. Beneyton, "A comparison of the mechanical stability of silicon nitride films deposited with various techniques," Applied surface science, vol. 260, pp. 69-72, 2012.

[6] H. Y. Wong, N. Braga, and R. Mickevicius, "AlGaN/GaN rake-gate HFET: A novel normally-off HFET based on stress and layout engineering," in Simulation of Semiconductor Processes and Devices (SISPAD), 2016 International Conference on. IEEE, 2016, pp. 61-64.

[7] W. Saito, Y. Takada, M. Kuraguchi, K. Tsuda, and I. Omura, "Recessedgate structure approach toward normally off high-Voltage AlGaN/GaN HEMT for power electronics applications," IEEE Transactions on Electron Devices, vol. 53, no. 2, pp. 356-362, Feb. 2006.

[8] Y. Cai, Y. Zhou, K. Chen, and K. Lau, "High-performance enhancementmode AlGaN/GaN HEMTs using fluoride-based plasma treatment," IEEE Electron Device Letters, vol. 26, no. 7, pp. 435-437, Jul. 2005.

[9] O. Hilt, A. Knauer, F. Brunner, E. Bahat-Treidel, and J. Wurfl, "Normally-off AlGaN/GaN HFET with p-type Ga Gate and AlGaN buffer," in 2010 22nd International Symposium on Power Semiconductor Devices IC's (ISPSD), Jun. 2010, pp. 347-350.

[10] L. Lucci, J.-C. Barbé, and M. Pala, "Full-quantum study of AlGaN/GaN HEMTs with InAlN back-barrier," in Simulation of Semiconductor Processes and Devices (SISPAD), 2015 International Conference on. IEEE, 2015, pp. 128-131.

[11] Synopsys Inc., CA, USA, "Sentaurus Process User Guide," 2015.

[12] K. Loiko, V. Adams, D. Tekleab, B. Winstead, X.-Z. Bo, P. Grudowski, S. Goktepeli, S. Filipiak, B. Goolsby, V. Kolagunta et al., "Multi-layer model for stressor film deposition," in Simulation of Semiconductor Processes and Devices, 2006 International Conference on. IEEE, 2006, pp. 123-126.

[13] Synopsys Inc., CA, USA, "Sentaurus Device User Guide," 2015.

[14] S. Strauss, A. Erlebach, T. Cilento, D. Marcon, S. Stoffels, and B. Bakeroot, "TCAD methodology for simulation of GaN-HEMT power devices," in 2014 IEEE 26th International Symposium on Power Semiconductor Devices IC's (ISPSD), Jun. 2014, pp. 257-260.

[15] I. Vurgaftman and J. R. Meyer, "Band parameters for nitrogen-containing semiconductors," Journal of Applied Physics, vol. 94, no. 6, p. 3675, 2003. 